\title{
Application of Computer Image Recognition Technology in Campus Monitoring
}

\author{
Tiejun Feng, Yuyou He
}

\author{
Computer Science, Zaozhuang Vocational College of Science Technology, Tengzhou, 277500 China.
}

\begin{abstract}
Based on the application of computer image recognition technology in university campus monitoring, to automatically realize the intelligent management of university by image recognition technology, this scheme could be used in the practical application of campus situation monitoring, face recognition, campus anomaly monitoring, fire prevention and theft, etc. In the process of intelligent automatic monitoring, we could make use of sound, light, electricity, mobile communication and so on for early warning; it could also analyses the behavior characteristics of university personnel, and automatically form monitoring reports and reports. Finally, the Java language was used to implement the algorithm, and the video and audio stream scheme FFmpeg was used to intercept the monitored video. The image processing was mainly called open source cross platform computer vision library, Opens, to decompose and recognize the face in the image. The simulation experiment results showed that the image recognition algorithm based on image fingerprint had short operation time and high efficiency. This method can effectively monitor and monitor the abnormal changes of campus, face recognition, statistical time and the number of colleges and universities, and can monitor and judge the accidents such as fire and theft.
\end{abstract}

Key words: Image Recognition; Intelligentization; Video Surveillance.

\section{INTRODUCTION}

It is of great significance to establish a set of monitoring and control system in the laboratory of University, but the function of the traditional monitoring system is relatively simple. It is limited to video recording, post playback, monitoring and control [1]. And it usually needs continuous and continuous manual monitoring, manual playback and search, low efficiency, thus it results in huge human waste. It can be seen that the traditional monitoring system cannot meet the needs of modern laboratory management.

According to China's security web site, surveillance personnel stare at the screen for more than minutes of missing video information and make it meaningless; in the London subway case, the security personnel took an hour to find the information needed on a large number of tapes. In the intelligent monitoring system, the monitoring picture is automatically analyzed from the image recognition module to the monitoring screen. Once the abnormal situation is analyzed, the alarm signal is automatically issued through the sound optoelectronic and other prompting, which leads people to monitor the people's attention, subverts the traditional mode of monitoring and analysis by the monitoring personnel and greatly alleviated the supervision and the burden of personnel. It is because the intelligent video surveillance has the advantages of digital, network and intelligence which cannot be compared with traditional artificial monitoring, and it is in line with the development trend of modern monitoring system [2], so the traditional video surveillance is being gradually replaced by intelligent video surveillance and is widely used in various industries.

In the last more than 10 years, the research of video surveillance has been developed in China, and the researchers at home and abroad have also carried out the research. For example, the Tsinghua University, Dr. Liu Xuedong and others, and have realized the intelligent monitoring system based on the automatic detection and 
classification of moving targets in order to solve the difficult problems of human body recognition under the complex background of outdoor. However, the design is too complex to be applied to real-time monitoring system. The image processing and Pattern Recognition Research Institute of Shanghai Jiao Tong University, Zhou Xiao's and others, realizes the image segmentation and recognition in the intelligent alarm system, but this design does not fully take into account the noise interference to the image, and the practicability is not strong. Wang Hoisin and Zhang Yan, the computer department of the Jinan University, Guangzhou, designed a kind of intelligent residential monitoring and theft protection system. This system is mainly used for intelligent alarm after illegal biological intrusion. It can only distinguish between animals and people, and it is easy to produce false alarm, and it is not universal. Professor Xin Shushing, of the Department of computer science and technology of Tsinghua University, has designed a system model algorithm [4], which uses real-time video image recognition technology to detect and statistics people with irregular behavior. The algorithm is applied to the Mao Zedong Memorial Hall in the year and can be used to count the number of people looking forward to each day in real time. In recent years, there have been some intelligent video surveillance products in the market, such as gold eye gold eye in Beijing, walker king of Beijing Walker and other products.

The intelligent video monitoring system not only conforms to the development trend of the industry, but also represents the future development direction of the monitoring system. It contains a huge market prospect and potential economic value [5], which is highly valued by the academic and industrial circles. Therefore, intelligent video surveillance has become a hot issue in the field of intelligent video information processing, and an important basic component of intelligent video surveillance is image recognition. It is a prerequisite for analysis and decision, and the result of image recognition determines the analysis results of video surveillance. There are a lot of researches on image recognition at present, but few of the images for real time monitoring in the laboratory are very few. The real-time laboratory monitoring requires that the image recognition speed is fast, the efficiency is high, the abnormal face recognition is good, the recognition needs to be combined with time and place and so on, but the image recognition is light and the background [6] is with low quality. The requirement is very low, and universal image recognition cannot adapt to real-time laboratory monitoring. Therefore, intelligent surveillance based on image recognition has important academic value and practical significance.

\section{LITERATURE REVIEW}

It is of great significance to establish a set of monitoring system in colleges and universities, but the function of the traditional monitoring system is relatively simple. It is limited to video recording, post playback, monitoring and control [7], and it usually needs continuous manual monitoring, manual playback and search, which is with low efficiency and results in huge human waste. It can be seen that the traditional monitoring system cannot meet the needs of modern university management.

\section{METHODOLOGY}

The image recognition algorithm of campus monitoring is applied to the practical applications such as scene monitoring, face recognition, scheduling, anomaly monitoring, fire prevention and anti-theft. In the process of intelligent automatic monitoring, early warning [8] can be made using sound, light, electricity and mobile communication, and the behavior characteristics of campus personnel can be analyses, and monitoring reports can be formed automatically. The monitoring video of the hour is analyses. Some functions can be timely early-warning and help the related personnel to monitor video. This requires the research algorithm to be fast and flexible. But considering the relatively stable monitoring environment of campus monitoring, the assistance and review of campus security can be ignored and the accuracy can be reduced in exchange for its time efficiency.

In the paper, we will focus on the most common human image monitoring in the laboratory, extracting the key area of the human image into the size of $8 * 8$ by the open source library function, with a total of 64 pixels. The role of this step is to remove the details of the picture, and it only keep the basic information such as structure, shade, and other basic information and abandons the different sizes and proportions of the picture difference, so as to facilitate the pre-processing of the picture again. Then it calculates the average grey value of all 64 pixels, which is as shown in formula one. 


$$
p v(i, j)=\sum_{i=1}^{8} \sum_{j=1}^{8} P(i, j) / 64
$$

When the average value of PV (i,j) is obtained, each pixel grey value $(i, j)$ of the key region image is compared with the mean value PV $(i, j)$, and the average value PV $(i, j)$ is greater than or equal to the average value of PV $(i, j)$. It is recorded as 1 and is recorded as 0 . As shown by the formula two and formula three.

$$
\begin{aligned}
& P_{(i, j)} \geq p v(i, j) \rightarrow \operatorname{com}(i, j)=1 \\
& P_{(i, j)}<p v(i, j) \rightarrow \operatorname{com}(i, j)=0
\end{aligned}
$$

\section{RESULTS AND DISCUSSION}

\section{The Realization and Analysis of the Change Rate of the Laboratory Monitoring Image}

The content of the experimental video showed that two people were in a laboratory experiment within onesixteen seconds, but they entered a strange person in sixteenth seconds. The purpose of the experiment was to detect unfamiliar people, record their heads, and automatically analyses the person's entry time and the image for sixteenth seconds when the video was analyses, and the image changed. The rate of conversion increases. It is worthwhile for managers to carry out video playback and further judgment. We use the system's lab monitoring image rate of change function to analyses the video and get the following analysis results, which is as shown in Figure 1.

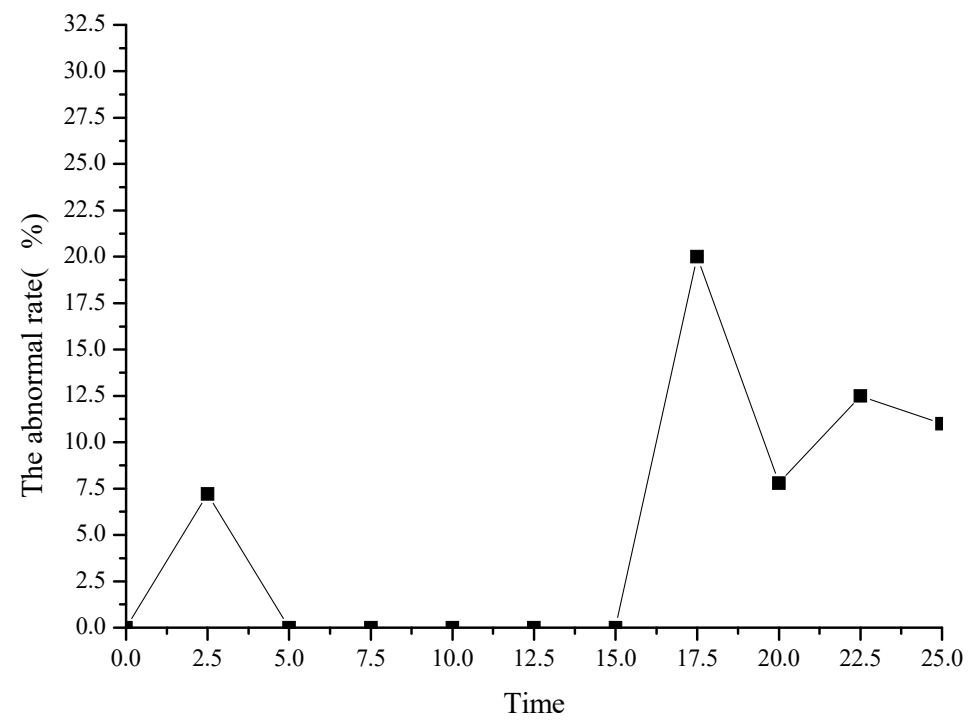

FIGURE 1. Image rate analysis results.

As shown in Figure 1, the results of the system are similar to the actual results. When the newcomers enter the laboratory in second, the rate of change in the laboratory increases immediately, and the change rate of the image tends to be stable when the newcomers sit down. This function can reduce the pressure of managers' real-time monitoring and enhance the pertinence of post playback. 


\section{Implementation and Analysis of Abnormal Statistics in Laboratory Monitoring}

In this function, we should not only detect the change of video, but also reflect the abnormal degree of laboratory video monitoring. To help laboratory managers to check the normal degree of monitoring in a certain period of time in real time or after the event, whether it belongs to the normal monitoring state, and whether there are abnormal situations. After setting the corresponding white list library and normal parameters, the video is analyses by using the system, and the result is shown in Figure 2.

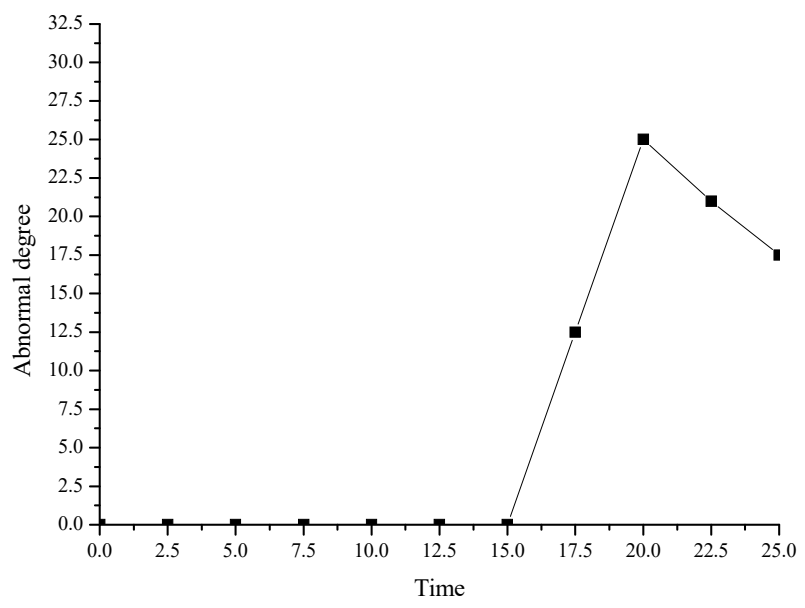

FIGURE 2. Anomaly analysis.

As shown in graph analysis, the exception is in an abnormal state after a second. After detecting the stranger entering, the system detects the person in the abnormal white list, increases the anomaly, and maintains a high anomaly with the presence of the person until the person leaves, or the administrator enters the white list.

\section{Realization and Analysis of Statistics of Face Abnormalities in Laboratory Monitoring}

The monitoring and analysis system extracts and analyses the image of the video captured in the video and compares the data in the white list library and the blacklist library. The results of the analysis are obtained, which are shown in Figure 2 below.

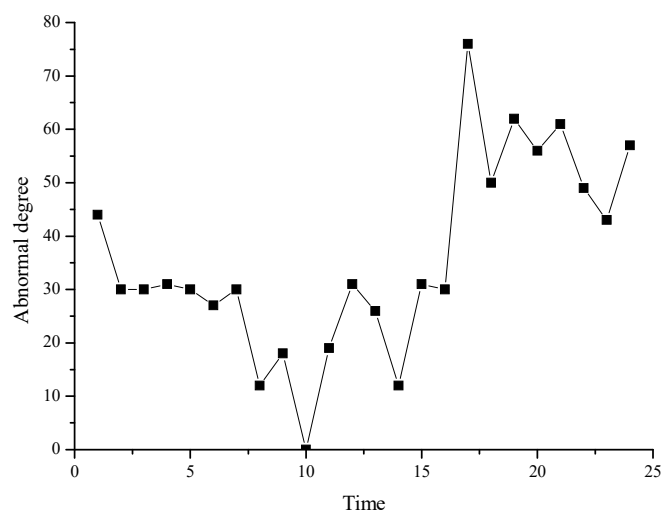

FIGURE 3. Face anomaly monitoring analysis. 
In this application, the system is more targeted to judge the abnormal degree of monitoring according to the face, and the analysis of the character anomaly is more accurate. From the result of analysis, the outlier is higher than the image analysis only, and the image of the different state of the abnormal face is recorded.

\section{The Realization and Analysis of the Early Warning of the Accident in the Laboratory}

The monitoring system can also be used for early warning of accidents, such as fire, theft, and so on. It can be early-warning by sound, light, electricity and mobile communication through the image monitoring and recognition at certain time (such as night, holiday), special location (window, balcony).

\section{CONCLUSION}

On the basis of the achievements of a large number of previous image recognition and intelligent video monitoring, the paper designed a video surveillance scheme for laboratory video monitoring in the laboratory. The main work is as follows: first, the achievements of previous research on image recognition and intelligent video surveillance are summarized. It can be divided into target detection, tracking and behaviour recognition, including motion detection, target classification, target tracking, behaviour recognition and other aspects. Secondly, according to the characteristics of laboratory monitoring, an image recognition algorithm based on image fingerprint is designed to adapt to the laboratory monitoring. It reduces the operation time of image recognition, improves the efficiency of image recognition, and lays the foundation for real-time intelligent monitoring. The image recognition algorithm based on image fingerprint includes five steps: extracting key areas, processing colour, grey value calculation, colour contrast and hash value calculation. The experimental results show that the speed and accuracy of the image recognition are improved accordingly. Thirdly, a laboratory monitoring scheme based on the image fingerprint matching algorithm and Opens is designed. The typical application of the monitoring scheme in the laboratory includes the following aspects: the analysis of the rate of change of the image, the statistics of abnormal degree of monitoring, the statistics of the degree of face anomaly, the statistics of the laboratory operation monitoring, the early warning of the accident. The algorithm and experiment system are implemented in Java language, and the main functions are expounded and analysed in detail. The simulation experiment results show that the image recognition algorithm based on image fingerprint has short operation time and high efficiency. This method can effectively monitor the abnormal changes in the laboratory, face recognition, operation time and the number of entry and exit laboratories and can be early warning of the fire.

\section{REFERENCES}

1. Oscar S, Invar V. The Monitoring System of an Actual Technical Condition for Pumping Units with Frequency Analysis. Procardia Engineering, 2017, 176, pp 144-149.

2. Long W, Xia L, Wang X L. A rapid automatic analyzer and its methodology for effective bentonite content based on image recognition technology, 2016, 13(5), pp 322-326.

3. Mancos P, Foliate a, Starvation a, et al. Accuracy evaluation of the optical surface monitoring system on EDGE linear accelerator in a phantom study. Medical Dosimeter Official Journal of the American Association of Medical Dosimetrists, 2016, 41(2), pp. 173-179.

4. Liu J H, Chen Y F, Lin T S, and et al. An Air Quality Monitoring System for Urban Areas Based On The Technology Of Wireless Sensor Networks. International Journal on Smart Sensing \& Intelligent Systems, 2017, 5(1), pp. 191-214.

5. Gers T, Holzäpfel F, French M, et al. The Wake Vortex Prediction and Monitoring System WSVBS Part II: Performance and ATC Integration at Frankfurt Airport. Air Traffic Control Quarterly, 2016, 17(2009), pp. 301322.

6. Xu B, Xu L, Cain H, et al. The design of an m-Health monitoring system based on a cloud computing platform. Enterprise Information Systems, 2017, 11(1), pp. 17-36.

7. Jian $\mathrm{D}$, Yao Z, Li C. The transformer winding temperature monitoring system based on fiber brag grating. International Journal on Smart Sensing \& Intelligent Systems, 2017, 8(1), pp. 538-560.

8. Fanzine A, Fiorucci M, Martino S, et al. Multi-sensor system designed for monitoring rock falls: The experimental test-site of Acute (Italy). Rendiconti Online Societal Geological Italian, 2016, 41(2016), pp. 147150. 